\title{
Intrauterine Spontanamputation an den oberen Extremitäten bei einem 5 Monate alten Fötus mit vollständiger Erhaltung des die Amputation be- dingenden Amniosfadens.
}

\author{
Von \\ Dr. Jacob Wolff, prakt. Arzt in Berlin. \\ (Mit 1 Abbildung im Text.)
}

Intrauterine Spontanamputationen sind schon seit langer Zeit den Aerzten bekannt. Schon Haller kannte dièses Vorkommniss. Allein die Ursache und die Art des Zustandekommens dieser Verstümmlung blieb lange Zeit in Dunkel gehüllt. Haller z. B. bezog diese Missbildung willkürlich auf gehemmte erste Bildung. Man glaubte zunächst, dass derartige Verstümmlungen durch Umschnürungen mittelst der Nabelschnur zu Stande kämen. Dann aber fand man an fast abgeschnürten Extremitäten noch kurze Fäden und Schlingen. Man deutete diese dahin, dass die Haut des Foetus in einem entzündlichen Zustande sich befunden hätte, und dass die Fäden als organisirte Lymphprodukte einer vorausgegangenen exsudativen Entzündung anzusehen seien (Montgomery) ${ }^{\mathbf{1}}$ ).

Frickhöfer $\left.{ }^{2}\right)$ z. B. deutete in dieser Weise einen von ihm beobachteten Fall von Selbstamputation mit noch anhaftenden kurzen Fäden und konnte aus der Literatur $z u$ dieser Zeit (1856) nur 3 Fälle dieser Art anführen.

1) Montgomery, Lehre von den Zeichen der Schwangerschaft. Uebersetzt ron Schwann. Bonn 1839.

2) Virchow's Archiv. Bd. X. S. 110.

Archiv f. Gynäkologie, Bd. 60. H, 2. 
282 Wolff, Intrauterine Spontanamputation an den oberen Extremitäten etc.

Aehnlich deutete Simpson ${ }^{1}$ ) diese Fäden.

Erst Gurlt ${ }^{2}$ ) und nach ihm Simonart ${ }^{3}$ ) brachten diese Fäden mit den Eihäuten in Verbindung. Letzterer glaubte, dass durch entzündliche Processe sowohl an den Eihäuten als auch am Fötus Verwachsungen entstehen, aus denen sich dann Bänder (Simonart'sche Bänder) entwickelten, und erst G. Braun ${ }^{4}$ ) suchte die vorgefundenen Spontanamputationen dadurch zu erklären, dass durch eine Faltung des Amnios mit nachträglicher Verlängerung durch Dehnung Fäden gebildet werden können, die dás weitere Hervorkeimen des schaufel- oder spatelförmigen embryonalen Auswuehses - also die Bildung der Extremitäten hindern, oder Theile davon ablösen, dass die neben abgelösten Gliedern sich vorfindenden Ligamente nicht als Entzündungsprodukt des Amnios, sondern als ein abnorm geformter Theil des Amnios selbst zu betrachten seien.

Es wurden dann wiederholt Missbildungen mit derartig intrauterin entstandenen Sclbstamputationen beobachtet; es glückte jedoch nie einen Zusammenhang der noch an den Gliedmaassen etwa vorhandenen Fäden mit dem Amnios nachzuweisen, da die meisten Beobachtungen an reifen geborenen Kindern gemacht wurden, bei denen natürlich derartige Fäden durch den Geburtsact zerrissen wurden.

Aber trotzdem gelang es auch nicht einmal in der Placenta, irgend welche Aufklärung durch Auffinden von Fäden im Amnios zu erhalten.

Erst Küstne ${ }^{5}$ ) konnte bei einem lebendgeborenen mit fötalen Amputationen an Händen und Füssen verstümmelten Kinde an der Placenta neben der Insertion des in einer bäutigen Scheide steckenden Nabelstranges einen etwa $2 \mathrm{~cm}$ langen Faden mit einem Träubchen, welches er als abgeschnürte Phalanx deutete, beobachten, welchen er als den die Verstümmelung verursachenden Strang anspricht.

Späterhin konnte Olshausen ${ }^{6}$ ) ebenfalls bei einem lebend geborenen Kinde mit 3 amputirten Fingern der linken Hand an der

1) Dublin. med. Journ. 1836. Vol. X.

2) Berl. med. Zeitung. 1833. No. 3.

3) Arch. de la Med. Belg. 1846.

4) Neuer Beitrag zur Lehre von den amniotischen Bändern. Wien 1862 .

5) Zeitschrift f. Geburtsh. u. Gynäkol. Bd. XX. S. 445.

6) Ebend. Bd. 34. S. 143. 
Wolff, Intrauterine Spontanamputation an den oberen Extremitäten etc. 283

Insertionsstelle des Nabels eine weisse Masse entdecken, welche sich als zusammengeballtes Amnios erwies, an welchem 4 bis 6 feine Fäden waren, welche die Amputation der Finger bewirkten.

Eine vollständige Erhaltung des abschnürenden Fadens ist bisher jedoch, soweit ich die Literatur durchgesehen habe, noch niemals beobachtet worden.

Die Erhaltung eines solchen Fadens ist natürlich nur dann möglich, wenn es gelingt, den mit solch' einer Verstümmlung behafteten Fötus in Zusammenhang mit seinen Eihüllen und der Placenta aus dem Uterus zu entfernen.

Ein derartiges Präparat habe ich nun vor einiger Zeit gewonnen, welches in selten deutlicher Weise die Herkunft der Fäden und die Art und die Phasen der intrauterinen Amputation veranschaulicht.

Frau D., Arbeiterfrau, 37 Jahre alt, seit 9 Jahren verheirathet, ist nie ernstlich krank gewesen. Menstruation regelmüssig. Lues nicht nachweisbar. Der Mann hat nur vor der Verheirathung an Gonorrhoe gelitten. Missbildungen sind weder in der Familie des Mamnes, noch der Frau, soweit erinnerlich, vorgekommen.

Im Jahre 1892 wurde ein Kind in normaler Weise geboren. In Jahre 1898 dann Abort im 2. Monat, den ich seiner Zeit ausräumte.

Im Jahre 1899 fand die letzte Periode am 3. Mai statt.

Am 12. Juni consultirte mich Frau D. Ich konnte die Gravidität feststellen, und da die Frau gerne ein Kind austragen wollte, rieth ich ibr dringend, "sich zu schonen.

Diesem Rathe kam die Frau auch etwa 21/2 Nonate lang nach. Da es ihr körperlich gut ging, glaubte sie wieder tüchtig mitarbeiten zu können, sie wusch wieder und hob schwere Körbe mit Wäsche. Seit dieser Zeit - also etwa seit Ende August - will Frau D. alle 4 Wochen blutigen Ausfluss gehabt haben, der nur einige Tage anhielt, und dem sie weiter keine Bedeutung beilegte, weshalb sie auch keine äratliche Hilfe in Anspruch nahm.

Von Nitte October an jedoch will sie sich unbehaglich gefühlt haben, - Kältegefühl im Kücken und Appetitlosigkeit stellten sich ein. Ausserdem fiel es ihr auf, dass ihr Leib nicht stärker wurde, so dass sie zur Vermuthung kam, sie wäre am Ende gar nicht schwanger.

Am 20. November stellten sich heftige Blutungen ein.

Als ich Fran D. am Nachmittage dieses Tages untersuchte, fand ich den Muttermund für 2 Finger durchgängig. Die Spitze des Ovums war in der Cervix zu fühlen. Tamponade.

Nachts um 1 Uhr erneute heftige Blutung. Entfernung der blutdurchtränkten Tampons. Das Ovum war zur Hälfte schon aus der Cervix ausgetreten.

Durch combinirte Compression von aussen und von der Scheide aus gelang es mir, das Ovum in toto in die Vagina hineinzupressen. Ich konnte constatiren, dass das $\mathrm{Ei}$ mit unversehrten schwappenden 
284 Wolff, Intrauterine Spontanamputation an den oberen Extremitäten etc.

Eihüllen in der Vagina vollständig gelöst lag. Allein bei dem Versuch, nun das sehr grosse Ovum aus der Scheide zu entfernen, rissen dié Eihäute, und es entleerte sich eine ziemliche Menge ungefähr 8-10 Esslöffel voll (da jegliche Assistenz mangelte, konnte ich eine genauere

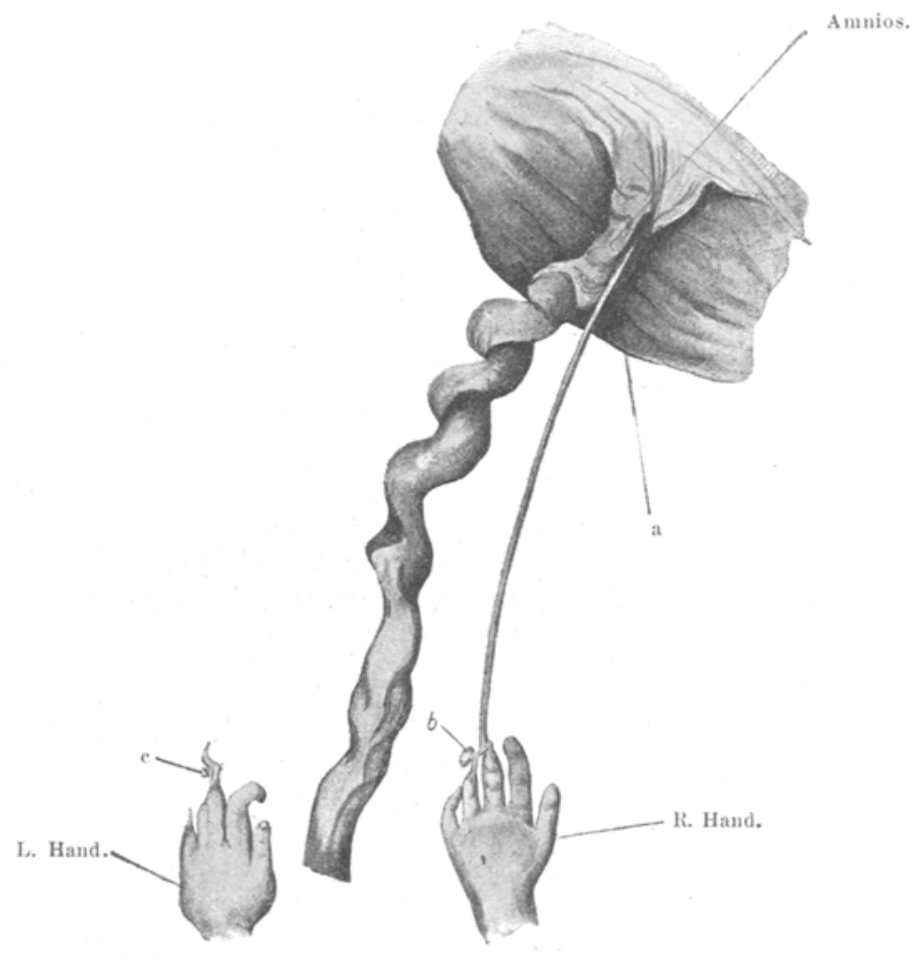

Messung nicht vornehmen) gelblich trüben Fruchtwassers. Den Fötus im Zusammenhang mit seiner Placenta und Eihüllen zu entfernen, gelang nun leicht.

An dem Fötus konnte ich num sofort die unten näher beschriebene Anomalie constatiren. Der Fötus selbst war abgestorben, die Haut an einzelnen Stellen schon etwas matsch, sodass man den Tod der Frucht auf etwa 4-6 Wochen zurückdatiren konnte.

Aus rein äusseren Gründen konnte ich das Präparat zunächst leider nur in ein Gefäss mit Brennspiritus legen. Ich liess das Präparat einige Tage später von Fräulein Paula Günther zeichnen, wobei Herr Prof. Langerhaus mit seinem Rathe gütigst zur Seite stand. (Bei der Zeichnung sind aus äusseren Gründen nur die Hände und der in Betracht kommende Theil der Placenta berücksichtigt worden.). 
Wolff, Intrauterine Spontanamputation an den oberen Extremitäten etc. 285

Die Beschreibung habe ich leider nur an dem durch den Spiritus ziemlich zusammengeschrumpften Präparat machen können. Die angegebenen Maasse sind infolge dessen kleiner, als sie in Wirklichkeit waren.

Maasse der Placenta: 13:131/2 cm. Die fötale Fläche ist glatt und vollständig vom Amnios bekleidet, welches überall unversehrt ist. An der einen Seite der Amnioshöhle befand sich ein haselnussgrosses, loses Blutcoagulum. Nach der Mitte der Placenta verdickt sich das Amnios zu einer wulstigen Scheide, welche die in $\mathrm{hr}$ entspringende etwa $17 \mathrm{~cm}$ lange Nabelschnur noch $2 \mathrm{~cm}$ hoch umkleidet. Die Insertion der Nabelschnur liegt ziemlich central. Auf der linken Seite ist die Nabelschnur mit dieser Amniostasche fest verwachsen, auf der rechten Seite befindet sich eine Lücke (a), aus deren Tiefe ein etwa $1 \mathrm{~mm}$ starker Faden entspringt, der bei seinem Austritt sich mit einem Zipfel des Amnios verbindet und nun als ziemlich starker gedrehter Faden zur rechten Hand des Fötus läuft. Die Länge dieses Fadens betrug etwa $10 \mathrm{~cm}$.im frischen Zustande.

Man konnte ziemlich kräftig an dem Faden ziehen, ohne dass er sich von seinen Insertionsstellen lockerte.

Das Präparat, welches etwa 10 . Stunden lang in Spiritus gelegen hatte, wurde dann in eine Formalinlösung gebracht. Hierbei faserte sich nun der Faden in mehrere einzelne Fäden auf. Der Zusammenhang zwischen Amnios und Fötus wurde am Präparate schliesslich wur noch durch einen ganz feinen Faden aufrecht erhalten.

An der rechten Hand hatte dieser Faden nun zunächst die Endphalange des Ringfingers, mit welcher er fest verwachsen war, abgeschnürt; doch ist die Phalange noch als kleines Knöpfchen (b) mit dem Finger im Zusammenhang geblieben. Vom Ringfinger verläuft der Faden zur Endphalanx des Mittelfingers, dessen Nagelglied umschnürt und verkümmert ist.

Die Finger der rechten Hand sind nirgends mit einander verwachsen. Der Daumen besitat einen normalen Nagel.

An der linken Hand finden wir eine Abtrennung von 2 Phalangen des kleinen Fingers, Syndactylie des Ring- und Mittelfingers. An Stelle der Endphalangen dieser beiden Finger befinden sich kurze Bänder und Fäden, an deren einem Ende beim Mittelfinger ein kleines Knöpfchen (c) sitzt (abgeschnürte und verkümmerte Phalange). 
286 Wolff, Intrauterine Spontanamputation an den oberen Extremitäten etc.

Die Abschnürung dieser Phalangen erfolgte höchst wahrscheinlich durch einen zweiten Faden, der gleichfalls aus der Lücke im Amnios neben der Insertion der Nabelschnur entsprang (auf der Zeichnung nicht angegeben), etwa $4 \mathrm{~cm}$ lang war und im unteren Drittel nach der Placenta zu ein Kölbchen trug (höchstwahrscheinlich eine abgeschnürte Phalanx).

Der Fötus ist etwa $24 \mathrm{~cm}$ lang und zeigt sonst nichts Anormales.

Nan hat num verschiedene Ursachen für die Entstehung dieser amniotischen Bänder angegeben. Wir haben schon oben die älteren Anschauungen von Simonart und Braun erwähnt.

Erst Küstner (l. c.) gab eine neue Entstehungsart an.

Er fand nämlich in seinem Fall, dass die die Nabelschnur an ihrer Insertionsstelle umhüllende Scheide an ihrer obersten Partie nur einen spiralig gedrehten Faden darstelle. Einige Cirkeltouren dieser Scheide waren zuerst angelöthet gewesen, späterhin aber frei schwimmend geworden. Die ganze fötale Oberfläche des Chorions entbehrte eines Amniosüberzuges, die Nabelschnur besass jedoch eine Amniosscheide, die jedoch so klein war, dass sie niemals die ganze Fläche des Chorion bekleiden konnte.

K. nimmt deshalb an, dass das Amnios auf einer niederen Wachsthumsstufe stehen geblieben ist und erklärt diesen Umstand in seinem Falle aus einem Trauma, den die Gravida im 2. Monat ihrer Gravidität erlitten hätte. Das Amnios wäre g erissen, der Riss nicht wieder geheilt, das Amnios hätte dann frei im Fruchtwasser flottirt und sich allmälig zu einem Strang gedreht unter Mitwirkung der Bewegungen des Fötus. Die Nabelschnurscheide sei um diese Zeit schon fest mit der Wharton'schen Sulze des Nabelstranges verwachsen gewesen: denn das Amnios hätte sich nur vom Chorion nicht aber von der Nabelschnur abgedreht. In diesem gedrehten frei flottirenden Strang hätten sich nun die äussersten Enden der Extremitäten des 2 Monate alten Fötus gefangen.

Für unseren Fall würde diese Deutung nun nicht zutreffend sein können, da die fötale Fläche des Chorion vollständig und glatt vom Amnios bedeckt war. Auch war die Ausdehnung der Amnioshöhle dem Alter des Fötus entsprechend genügend gross. Das in der Amnioshöhle befindliche Blutcoagulum und die Blutungen unserer Patientin während der Gravidität könnten viel- 
Wolff, Intrauterine Spontanamputation an den oberen Extremitäten etc. 287

leicht zu Gunsten einer solchen Theorie gedeutet werden, aber es liess sich in unserem Falle in keiner Weise eine solche Wachsthumsstörung des Amnios durch einen Riss nachweisen.

Auch Olshausen (l. c.) glaubt, dass in seinem Falle die amniotischen Fäden durch einen Riss des Amnios entstanden seien. Das Amnios, welches bekanntlich ja in der allerersten Zeit dem Fötus direct anliegt, sich dann durch Absonderung von Fruchtwasser vom Fötus abhebt und sich an das Chorion anlegt, könnte durch geringes Fruchtwasser in seiner Annäherung an das Chorion behindert werden; dadurch wïre es dem Fötus möglich gewesen, das Amnios zu zerreissen.

Aber auch diese Erklärung wäre für unseren Fall nicht hinreichend. Wie wir schon oben erwähnt haben, war in dem Orum eine ziemlich normale Menge von Fruchtwasser vorhanden, wenn wir leider auch nicht ein genaues Maass angeben können.

Winckel1) meint, dass selbst bei Anhäufung von Blut und Plüssigkeit zwischen Chorion und Amnios das letztere, da es ja die allerfesteste Eihaut und fester wie die Nabelschnur ist, trotz bedeutender Abhebung nach innen unzerrissen bleiben kann. Denkt man sich nun nach $W$. eine solche und die Abhebung des Amnios wie bei der Geburt des Kindes in dem intacten Amnios, so könnte eine Falte desselben bis zur Nabelschnur rücken. Fände alsdann eine Berstung nach 2 Seiten in die Amnioshöhle statt, so könnte ein ganz kleiner Embryo eine solche Oeffnung allenfalls passiren, und aus der durchbohrten Platte bez. Falte würde dann eine Schlinge.

Eine andere Erklärung für die Entstehung der amniotischen Fäden gaben in allerjüngster Zeit Opitz und Graf Spee ${ }^{2}$ ):

Man hatte bisher angenommen, dass die Ammioshöhle dadurch entsteht, dass die rings um diẹ Fötalanlage durch Einsenken derselben in die Keimblase gebildeten Wülste (vordere Kopffalte -späterhin die seitlichen und hinteren Amniosfalten) sich über den Rücken der Keimscheibe erheben und schliesslich sich vereinigen.

Graf Spee vertritt nun die Ansicht, dass die Amnioshöhle sich ähnlich wie bei den Nagern mit Blätterumkehr als eine Flüssigkeitsansammlung in der vorher eine solide Zellkugel darstellenden Fötalanlage über der Rückenfläche des Embryo sich

1) Münchener med. Wochenschr. 1896. No. $17 \mathrm{ff}$.

2) Cfr. Verhandl. d. Gesellsch. f. Geburtsh. vom 24. 3. 1899 zu Berlin. 
288 Wolff, Intrauterine Spontanamputation an den oberen Extremitäten etc.

bildet, also von Anfang an einen geschlossenen Hohlraum darstelle.

Dadurch, dass die zuerst über die Rückenfläche gekrümmte Embryoanlage sich abflacht und dann über die Bauchhöhle sich krümmt, wird der Embryo gewissermassen in die Amnioshöhle hineingedrängt und diese auf die Bauchseite herumgezogen.

Man kann nach Ansicht des Grafen Spee sich schwer vorstellen, wie amniotische Fäden sich durch Vereinigung der Amniosfalten bilden können; wenn aber das Amnios sich als eine Flüssigkeitsansammlung in einer vorher soliden Zellkugel anlegt, so ist es leicht begreiflich, dass einmal Verbindungen, die die Amnioshöhle durchziehen, stehen bleiben können, wenn der Zusammenhang zwischen den Zellen fester ist, als dass er durch den Druck der sich zuerst zwischen den Zellen ansammelnden Amniosflüssigkeit aufgehoben werden könnte.

Derartig stehengebliebene Verbindungen können sehr wohl zum Hinderniss für die Ausbildung z. B. der fötalen Gliedmaassen werden, wenn sie sich gerade an der Stelle befinden, aus der dieselben hervorsprossen.

Man sähe deshalb nie amniotische Fäden vom Rücken des Embryo ausgehen; denn gerade über der Rückenfläche beginne die Trennung der Bestandtheile der Fötalkugel durch das Amnioswasser und schreite von dort nach den Seiten fort.

Wie weit diese Entstehungsursache der amniotischen Fäden auf unseren Fall zutreffend ist, lasse ich dahingestellt.

Es lässt sich in unserem Falle mit Bestimmtheit direct nachweisen, dass die Abschnürung der Phalangen durch ein amniofötales Band herbeigeführt worden ist. Man unterscheidet bekanntlich noch amnio-amniotische und föto-fötale Bänder.

Die Wülste des Amnios an der Insertion der Nabelschnur lassen vielleicht die Deutung zu, dass es sich hier um einen entzündlichen Process am Amnios gehandelt hat, insofern kehren wir wieder zu der alten Anschauung (Simonart's) zurück. Und eine Verwachsung zwischen Fötus und Amnios kann ja dadurch begünstigt werden, dass die Innenfläche des Amnios ebenso wie die Oberfläche des Embryo vom Ectoderm bekleidet ist. Ob nun ausserdem noch das Amnios einen kleinen Riss erlitten hat, (Lücke an der Insertion der Nabelschnur) und ein Zipfel dieses Risses mit zu einem Faden ausgezogen worden ist, lässt sich nicht 
von der Hand weisen, da wir ja gesehen haben, dass der aus der Tiefe der Tasche entspringende Faden sich direct mit einem Zipfel des Amnios vereinigt.

Die Drehung und Ausdehnung des Fadens spricht dafür, dass die Verwachsung frühzeitig, jedenfalls vor Absonderung des Fruchtwassers stattgefunden haben muss, da ja sonst die Flüssigkeit den Contact des Fötus mit dem Amnios hätte hindern müssen.

Für die Stärke dieser Fäden spricht der Umstand, dass trotzdem z. B. am unteren Drittel des zweiten oben beschriebenen Fadens sich bereits eine abgeschnürte Phalange befindet, der Faden, der nur noch mit einer ganz feinen Insertion an der linken Extremität hatte haften können, dem Zuge des sich immer weiter abhebenden Fötus doch noch um mehrere Centimeter hatte folgen können, ehe er riss.

Die Abschnürung der Phalangen an der linken Hand ist also schon sehr frühzeitig erfolgt. Hätte der Fötus seine vollständige Reife erlangt, dann wäre jedenfalls die kleine abgeschnürte, an dem Faden noch haftende Phalangelängst resorbirt worden.

Man kann sich nicht recht vorstellen, wie es möglich ist, dass eine kleine Amniosfalte an einer so minimalen Haftfläche, wie sie die Extremitätenanlage beim Embryo in frühester Zeit darstellt, zumal, wie in unserem Falle nur an den äussersten Phalangen, haften und dem ziemlich starken Zuge des sich abhebenden Fötus hätte folgen können, wenn nicht eine feste Verlöthung zwischen der Extremitätenanlage des Fötus und dem Amnios stattgefunden hätte, und solch eine innige Verwachsung kann wohl nicht durch einen rein mechanischen Vorgang, sondern höchstwahrscheinlich nur durch einen entzündlichen Process eintreten.

Anders liegt die Sache bei Einschnürung von Fötaltheilen durch einen amnio-amniotischen Faden, in welchem Falle also der abschnürende Faden seine beiden Haftpunkte im Amnios selber hat, also eine Schlinge um einen Fötaltheil bildet, welche durch den Zug des Fötus immer fester um den abgeschnürten Theil gezogen wird. Hierbei ist ein rein mechanischer Vorgang im Spiel.

Solche Amniosfäden und -schlingen können nun die schwersten Missbildungen beim Foetus hervorrufen, wie sie von den Autoren vielfach beschrieben worden sind, deren Erörterung an dieser Stelle nicht angängig ist. Diese Fäden schnüren jedoch 
290 Wolff, Intrauterine Spontanamputation an den oberen Extremitäten etc.

nicht nur Körpertheile ab, sondern können auch Spaltungen hervorrufen ${ }^{1}$ ). (Verdoppelung an Fingern).

Dass aber auch das Amnios selber pathologischen Processen unterworfen wird, ist uns aus mannigfachen Erkrankungen desselben bekannt.

Ich erinnere nur an das Hydramnios und an die papillären Wucherungen²), welche als. Auflagerungen bindegewebig mit dem Amnios verwachsen sind.

Es können also auch leieht Verwachsungen von Fötaltheilen mit solchen papillären Wucherungen entstehen, welche durch Dehnung zu Fäden ausgezogen werden können.

Ich möchte zum Schluss nicht unerwähnt lassen, dass in unserem Falle die Gravida behauptete, sie hätte sich im Beginne ihrer Gravidität sehr über einen Mann, der eine verkrüppelte Hand gehabt hätte, erschrocken; sie hatte diese Verstümmelung während ihrer ganzen Gravidität immer vor Augen gehabt und glaubt sich „versehen" zu haben. Der Ehemann bestätigte mir den Vorgang.

Ich möchte in unserem Falle, wo wir es mit mechanischen Vorgängen zu thun haben, diese Aetiologie nicht näher erörtern. Ich habe an einer anderen Stelle ${ }^{3}$ ) diesen Punkt ausführlicher behandelt.

1) Ahlfeld, Missbildungen der Menschen. Leipzig 1880.

2) Franqué, Zeitschr. f. Geb. u. Gynäk. 1897. Bd. 37.

3) Casuistischer Beitrag zur Lehre vom Versehen der Schwangeren nebst Bemerkungen über Hyperdactylie. Deutsche Med.-Zeitg. 1894. No. 50/51. 